\title{
Cobalt cardiomyopathy: clinical aspects
}

\author{
Yves Morin, André Têtu, and Gaston Mercier \\ From the Department of Medicine, Laval University, Quebec, Canada
}

On 24 August 1965 a 39-year-old stevedore was admitted to the Department of Surgery of the Hôtel-Dieu de Québec. The clinical picture was unusual. The patient complained of severe epigastric pain, with tenderness localized to the upper part of the abdomen. He was deeply cyanotic; this greyish hue was found especially in the face, neck, and abdomen. He was short of breath but lay flat in bed. Above all, he was very anxious and agitated, but did not present any evidence of incoherence or gross tremor. The patient admitted to drinking regularly an average of 25 pints of beer (Brand XXX) daily. On physical examination of the cardiovascular system his heart rate was regular at $I I 5$ beats per minute, and blood pressure was $80 / 60 \mathrm{~mm}$. $\mathrm{Hg}$. Arterial pulses were symmetrical but of small volume. The jugular veins were distended, and a prominent ' $a$ ' wave could easily be detected. Palpation of the praecordium was negative. No murmurs could be heard on auscultation of the heart, but loud third and fourth heart sounds were obvious.

In spite of the low blood pressure and the quiet heart, a diagnosis of beriberi was made. The patient was catheterized after he had been fully digitalized and received diuretics. Instead of the high cardiac output that was expected, the cardiac index was $1.81 . / \mathrm{min}$. and did not rise after a 25-second exercise period. The end-diastolic ventricular pressures were elevated in both ventricles. It was felt that this patient presented an unusual form of alcoholic myocardiopathy. He was treated in the usual fashion, given thiamine in large dosage, made a slow recovery, and went back to work, and to beer.

\section{Further cases}

In the next three weeks two identical cases were seen in the same hospital and three in other centres of the city. Seven cases were admitted in October, six in November, seven in January, eight in February, six in March, and finally four in the first days of April. All cases were found in the Quebec City area, and none were reported in other parts of the country.
There were 48 men and two women as patients. Most of them were middle-aged. Some men were unemployed. However, most were employed as truck drivers, waiters, dock workers, and construction workers. Although this was not a constant finding, many performed heavy physical labour.

All of these patients were unusually heavy beer drinkers, and 36 had been drinking heavily for at least 20 years. The average alcoholic intake was 24 pints daily, but one patient, a truck driver, drank an average of 20 quarts of beer daily. Most patients drank beer exclusively, but six occasionally consumed other types of alcoholic beverages. Although the average patient preferred draught beer sold exclusively in taverns, bottled beer was also consumed, since taverns are closed on Sunday in Quebec City.

By November it became obvious, and this was confirmed by a governmental epidemiological survey, that all of the surviving patients who could be questioned drank preferably (but not exclusively) Brand XXX. The XXX Brewery is in an unusual position in Quebec City. It was established on the site of the oldest brewery in North America, built by Jean Talon in 1668 to help combat alcoholism among French settlers. Its excellent-tasting brew was (and still is) very popular in Quebec and accounts for approximately 80 per cent of the local market. A beer of the same name is manufactured in Montreal. As far as could be ascertained, the only difference between the two products at that time was that the Quebec Brand XXX beer contained approximately ten times more cobalt sulphate. This chemical has been added to some Canadian beers since July 1965 to improve the stability of the foam. For this reason it has been added in larger breweries only to draught beer. However, in smaller breweries, such as in Quebec City, separate batches were not brewed for bottle and for draught beer; therefore the cobalt sulphate was added to all the beer processed in the XXX Brewery. Thus the intake of this food additive was increased to much higher levels than in Montreal.

Most patients were in good health from 
three months up to two weeks before admission. The first symptoms to appear were gastrointestinal. Every patient questioned admitted to a severe anorexia that prevented food intake and frequently limited beer consumption. Nausea and vomiting were frequent, and haematemesis was seen on seven occasions. Diarrhoea was often encountered, and there were four instances of melaena. Progressively signs of heart failure appeared: shortness of breath, dry and persistent cough, thoracic and right upper quadrant abdominal pain, often very severe, and ankle oedema.

On physical examination most remarkable signs were related to the cardiovascular system: a peculiar cyanosis, limited to the face and trunk, was typical enough to permit a spot diagnosis of the condition. The fact that melanin pigment was found to be increased on skin biopsy of these patients may in part explain this unusual coloration. The heart rate was always rapid but regular; this is in contrast with other types of primary myocardiopathy, in which arrhythmias, such as atrial fibrillation, are commonly encountered. The blood pressure was low, and arterial pulses were of small volume. Arterial thrombi (or emboli) were clinically encountered in five cases and were the cause of death in two of these. Hepatomegaly, ankle oedema, and jugular vein distension were seen in approximately half of the cases. Frequently a giant ' $a$ ' wave was recorded on the jugular phlebogram. The praecordium was quiet.

On auscultation of the heart, murmurs of mitral and tricuspid insufficiency, often heard in more common forms of primary myocardiopathy, were rare in this disease. Third and fourth heart sounds were frequently heard, however, and after they had disappeared with clinical improvement they could often be heard for several weeks after vigorous exercise. Pulmonary rales were heard in 27 out of 50 cases, and nine cases were admitted in frank pulmonary oedema.

\section{Mortality}

Of the 50 cases, 20 died. In these cases evolution was rapid, and the clinical descriptions fit the picture of Shöshin beriberi (Platt, 1958) with the exception that signs of hyperkinetic circulation such as 'the powerfully undulating pulsations in the heart region, the epigastrium and the neck' and the 'full arterial pulse' were not seen. However, the severe dyspnoea, the anxiety and restlessness, the epigastric and praecordial pain, the obvious cyanosis, and the auscultatory findings were similar. The patients died quickly, usually within 24 hours, generally in shock that did not respond to vasoactive amines, steroids, digitalis, or thiamine.

Haematological studies performed in these patients showed a haemoglobin or red blood cell count in the high normal range, and nine patients were frankly polycythaemic. Four patients tested had increased blood volume. Sedimentation rate was normal in all cases. Generally, serum iron levels were low in the face of high haemoglobin. Serum electrolytes - sodium, potassium, chloride, calcium, phosphorus, and magnesium - were usually within normal limits when studied; 14 cases out of 23 tested showed lactic acidosis, with a low $\mathrm{pH}$ generally indicating a poor prognosis.

Liver function tests were done in the majority of cases. Serum bilirubin was elevated in 23 out of 33 cases. Prothrombin time was prolonged in all patients tested, over twice control values in 16 out of 34 cases. This is somewhat surprising if one considers the frequent thrombotic complications encountered in this disease. Flocculation tests (thymol flocculation, thymol turbidity, and cephalincholesterol flocculation) were generally normal. Electrophoresis of serum proteins showed little disturbance: there was slight hypoalbuminaemia and an important elevation of serum globulins.

The serum enzyme levels showed striking elevations, and when performed during the acute stage of the illness confirmed the suspected diagnosis. Seven patients in shock in whom serum glutamic oxaloacetic transaminase was measured had levels above 2000 units. Other serum enzymes were also found to be elevated (glutamic pyruvic transaminase, lactic dehydrogenase, ornithine carbamyl transferase, isocitric dehydrogenase, aldolase). The elevation of these serum enzyme levels correlated well with the central hepatic necrosis found in the severe form of the disease and, especially in view of the high lactic dehydrogenase/bicarbonate dehydrogenase ratio, and of the disproportionately elevated levels of serum ornithine carbamyl transaminase, it was felt that these unusually high serum enzyme levels were hepatic and not myocardial in origin.

Attempts to measure thiamine in the blood were not successful. Thiamine deficiency was demonstrated in 13 patients tested by one of two methods: either by comparison of the expected with the actual levels of serum pyruvic acid in relation to serum lactic acid levels, or by the pyruvic acid tolerance test using a loading dose of glucose. One must interpret these results with caution, however, since thiamine deficiency, as detected by the erythrocyte transketolase determination, can be 
found in fasting subjects within four days (Haro, Brin, and Faloon, 1966). Virological and immunological studies were negative. Search for toxic substances such as arsenic or lead was negative.

\section{Electrocardiography and radiography}

Electrocardiograms were performed, at least once, in 48 out of 50 patients. Electrocardiographic tracings were remarkably similar in all cases in the acute stage as well as during the later stages of the disease. Tachycardia was the rule. Arrhythmias, all of them supraventricular, were seen in only three cases and then were transitory. This is, of course, in contrast to findings in other types of cardiomyopathy. Low voltage of the QRS segment in the limb leads was a constant finding, these waves not exceeding 5 millivolts even in cases in which pericardial effusion was shown not to be present by cardiac catheterization. These minicomplexes progressively disappeared and within a month were generally replaced by evidence of left ventricular hypertrophy.

Abnormalities in the $\mathbf{P}$ wave were bizarre and frequent and led to heated arguments as to whether right or left atriomegaly was present. Although all types of anomalies were seen, the most frequent finding was a broadpeaked $P$ wave with right axis deviation in the limb leads with a diphasic (plus and minus) $P$ wave in leads $V_{I}$ and V2. These abnormalities also disappeared within a month.

Intraventricular conduction defects were rarely seen. There were three cases of bundlebranch block. However, septal depolarization was felt to be abnormal in 22 cases. $Q$ waves were absent in left epicardial leads, and Q-S waves were seen in leads VI and V2. This pattern cleared up in two weeks.

Elevations of the ST wave were frequently seen in the right praecordial leads, and nonspecific $T$ wave anomalies were the rule. With time the $T$ waves became frankly negative, symmetrical, and 'ischaemic' in type. This last anomaly, in conjunction with high voltage of the QRS segment in limb and praecordial leads, remained stable for two to three months. At that time the QRS voltage decreased to normal and the $T$ waves in leads $V_{4}$ to V6 were simply flattened. After six to nine months 17 out of 22 cardiograms could be interpreted as normal.

Chest radiographs were taken in 46 patients, many of them with a portable machine. No specific pattern could be detected. All patients showed an enlarged heart to a varying degree, and all cavities were dilated, right and left. There was distension of the superior vena cava, and the more severe it was the graver the prognosis. The pulmonary arteries were dilated in half of the cases, and passive congestion of lung fields could be detected in the same proportion of patients. Pleural effusion was found in 12 cases, and in the two patients in whom cardiac catheterization, by revealing an abnormal distance between the catheter tip and the heart shadow border, suggested pericardial effusion, a cardiac angiogram was taken by injecting contrast material in the right atrium to confirm the diagnosis. With time the pulmonary congestion disappeared, the heart size decreased, and, six to nine months after the onset of the illness, heart size had returned to normal.

\section{Haemodynamic studies}

Haemodynamic studies were performed in 13 patients within 48 hours of admission. At the time of examination most patients had received digitalis and diuretics but not thiamine. Cardiac outputs were normal or low and did not increase with exercise or administration of isoproterenol. These figures were not significantly different from the cardiac index values of 136 cases of primary myocardial disease reported in the literature (Massumi et al., 1965; Goodwin et al., 1961; Fowler, Gueron, and Rowlands, 196I ; Sackner et al., 1961; Storstein, 1964; Yu et al., 1964; Dye et al., 1963; Boyd et al., 1965; Goodwin, 1964; Pietras et al., 1965; Wendt et al., 1962; Bourdarias et al., 1966). However, these results are significantly different $(p<0.05)$ from the cardiac index values of 12 cases of beriberi heart disease reported by Wagner (1965). As expected, pressures in the right ventricle showed an elevated systolic pressure, indicating increased pulmonary resistance developing as a consequence of an increased left end-diastolic pressure. The right ventricular pressure curves also indicated a decrease in ventricular compliance, as the ratio of end-diastolic pressure to systolic pressure was higher than $\mathrm{I}: 3$. Left ventricular pressure curves showed a low systolic pressure that was constant in the acute stage of the illness and indicated the low cardiac output in the face of normal systemic arterial resistance. However, as indicated previously, end-diastolic pressure was elevated, often exhibiting an 'atrial kick'.

In summary, these haemodynamic studies show a normal or low cardiac output, diminished myocardial compliance, absence of myocardial response to exercise or catecholamine, and a normal peripheral resistance. The evolution of these cases was variable, and it is doubtful whether therapy had much influence on the eventual outcome. Most patients were 
treated with digitalis, diuretics, and thiamine. Recovery was slow and progressive.

\section{Follow-up}

After discharge from hospital, patients were seen fairly regularly, although 8 were lost to follow-up. The remaining subjects failed to follow diet or to take their medication regularly; most important of all, 16 out of 22 returned to their drinking habits, consuming as much beer and of the same brand as they did before. Their food habits were identical. Many of them were inebriated when seen on follow-up studies. In spite of this, none had symptoms or physical signs referable to the cardiovascular system. Electrocardiograms were either normal or, in 5 cases out of the 22, showed minor and nonspecific $T$ wave changes. Chest radiographs were all within normal limits.

Thus between August 1965 and April 1966 in the Quebec City area a syndrome with definite clinical and haemodynamic features appeared. Similar cases were not seen in other parts of the country. However, one of the authors had the opportunity to examine cases in Omaha, Neb., and in Minneapolis, Minn. Other series probably exist elsewhere.

Clinically the patients presented as cases of severe heart failure with shock, liver involvement, gastrointestinal ulcerations, and thrombotic phenomena. There is a certain similarity between this disease and beriberi heart disease. In the latter, however, an abnormally high cardiac output with very low systemic resistance is a constant finding. This phenomenon would appear to be only one aspect of the disease, and myocardial involvement of the type observed in the Quebec myocardosis also has been found in beriberi (Akbarian, Yankopoulos, and Abelmann, 1966).

The aetiology of this syndrome will not be definitely established until the disease is experimentally reproduced in animals. However, we feel that the epidemiological, clinical, and pathological evidence is at present strong enough to incriminate cobalt as one of the essential causative factors of this syndrome. The toxicity of cobalt has been well studied at a clinical (Caujolle and Meynier, 1959) and at a cellular level (Dingle et al., I962), but a similar syndrome has not to our knowledge been reported previously. The fact that cobalt, as an antianaemia agent, has in the past been used extensively at much higher dosage (24 pints of Brand XXX beer would officially contain $8 \mathrm{mg}$. of cobalt sulphate, while 4 tablets daily of Roncovite would represent an intake of $60 \mathrm{mg}$. of cobalt chloride) without produc- ing such a dramatic picture would tend to indicate that other factors are at play to increase the susceptibility of these patients to cobalt. The nature of these other factors is the object of our investigation now.

\section{References}

Akbarian, M., Yankopoulos, N. A., and Abelmann, W. H. (1966). Hemodynamic studies in beriberi heart disease. American fournal of Medicine, 41, 197.

Bourdarias, J. P., Ourbak, P., Penther, P., Scebat, L., and Lenègre, J. (1966). Etude hémodynamique des myocardiopathies non obstructives d'origine indéterminée. Acta Cardiologica, 21, 285.

Boyd, D. L., Mishkin, M. E., Feigenbaum, H., and Genovese, P. D. (1965). Three families with familial cardiomyopathy. Annals of Internal Medicine, 63, 386.

Caujolle, F., and Meynier, D. (1959). Sur la toxicité du cobalt. Revue de Pathologie Générale et de Physiologie Clinique, 59, 245.

Dingle, J. T., Heath, J. C., Webb, M., and Daniel, $M$. (1962). The biological action of cobalt and other metals. II. The mechanism of the respiratory inhibition produced by cobalt in mammalian tissues. Biochimica et Biophysica Acta, 65, 34.

Dye, C. L., Genovese, P. D., Daly, W. J., and Behnke, R. H. (1963). Primary myocardial disease. II. Hemodynamic alterations. Annals of Internal Medicine, 58, 442.

Fowler, N. O., Gueron, M., and Rowlands, D. T., Jr. (196I). Primary myocardial disease. Circulation, 23, 498.

Goodwin, J. F. (1964). Cardiac function in primary myocardial disorders. British Medical fournal, I, 1527,1595 .

-, Gordon, H., Hollman, A., and Bishop, M. B. (I96I). Clinical aspects of cardiomyopathy. British Medical fournal, $1,69$.

Haro, E. N., Brin, M., and Faloon, W. W. (1966). Fasting in obesity. Thiamine depletion as measured by erythrocyte transketolase changes. Archives of Internal Medicine, $117,175$.

Massumi, R. A., Rios, J. C., Gooch, A. S., Nutter, D., De Vita, V. T., and Datlow, D. W. (I965). Primary myocardial disease. Report of fifty cases and review of the subject. Circulation, 31, I9.

Pietras, R. J., Meadows, W. R., Fort, M., and Sharp, J. T. (1965). Hemodynamic alterations in idiopathic myocardiopathy including cineangiography from the left heart chambers. American fournal of Cardiology, 16, 672 .

Platt, B. S. (1958). Clinical features of endemic beriberi. Federation Proceedings, 17, Suppl. $2,8$.

Sackner, M. A., Lewis, D. H., Robinson, M. J., and Bellet, S. (I96I). Idiopathic myocardial hypertrophy. American fournal of Cardiology, 7, 714.

Storstein, O. (1964). Primary myocardial disease. Acta Medica Scandinavica, 176, 731.

Yu, P. N., Cohen, J., Schreiner, B. F., Jr., and Murphy, G. W. (1964). Hemodynamic alterations in primary myocardial disease. Progress in Cardiovascular Disease, 7, 125.

Wagner, P. I. (1965). Beriberi heart disease: physiologic data and difficulties in diagnosis. American Heart fournal, 69, 200.

Wendt, V. E., Stock, T. B., Hayden, R. O., Bruce, T. A., Gudbjarnason, S., and Bing, R. J. (I962). The hemodynamics and cardiac metabolism in cardiomyopathies. Medical Clinics of North America, 46, 1445. 